\title{
Acute hypoglycemia results in reduced cortical neuronal injury in the developing IUGR rat
}

\author{
Anne M. Maliszewski-Hall', Ariel B. Stein ${ }^{1}$, Michelle Alexander ${ }^{1}$, Kathleen Ennis ${ }^{1}$ and Raghavendra Rao'
}

\begin{abstract}
BACKGROUND: Hypoglycemia $(\mathrm{HG})$ is common in intrauterine growth restricted (IUGR) neonates. In normally grown (NG) neonatal rats, acute HG causes neuronal injury in the brain; the cerebral cortex is more vulnerable than the hippocampus (HPC). We hypothesized that the IUGR brain is less vulnerable to HG-induced injury while preserving regional variation in vulnerability.
\end{abstract}

METHODS: We induced IUGR via bilateral uterine artery ligation on gestational day 19 (term 22 d) rats. On postnatal day 14, insulin-induced $\mathrm{HG}$ of equivalent severity and duration (blood glucose $<40 \mathrm{mg} / \mathrm{dl}$ for $240 \mathrm{~min}$ ) was produced in IUGR and NG (IUGR/HG and NG/HG). Neuronal injury in the cortex and HPC was quantified 6-72 h later using Fluoro-Jade B (FJB) histochemistry. The mRNA expression of monocarboxylate transporters, MCT1 and MCT2, and glucose transporters, GLUT1 and GLUT3, was determined using quantitative PCR.

RESULTS: There were fewer FJB-positive (FJB+) cells in the cortex of IUGR/HG; no difference was observed in FJB+ cells in HPC. Core body temperature was lower in IUGR/HG compared with NG/HG. MCT2 expression was increased in the IUGR cortex.

CONCLUSION: HG-induced neuronal injury is decreased in the cortex of the developing IUGR brain. Adaptations including systemic hypothermia and enhanced delivery of alternative substrates via MCT2 might protect against HG-induced neuronal injury in IUGR.

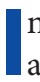
nfants born with intrauterine growth restriction (IUGR) as a result of placental insufficiency are at an increased risk for hypoglycemia (HG) in the neonatal period due to decreased glycogen stores, increased brain-to-body weight ratio, and an increased sensitivity to insulin $(1,2)$. It is unknown whether IUGR infants are at an increased risk for hypoglycemic brain injury. Neurodevelopmental deficits have been described in school-aged children who were born IUGR with respect to learning and memory, cognition, attention, and behavior, suggesting functional deficits in the cerebral cortex and hippocampus (HPC) (3-6). The cerebral cortex and HPC are particularly vulnerable to hypoglycemic brain injury in both humans and rodents (7-9) with the cortex being more susceptible to injury than the HPC in the developing rat brain $(7,10,11)$.

Animal models of IUGR describe altered carbohydrate and energy metabolism, blunted glucoregulation, and ineffective ketone body response to HG $(3,12-14)$ suggesting that the IUGR brain may be at greater risk of injury during periods of low glucose availability. Conversely, there is evidence that fetal metabolic adaptations as a result of IUGR might be protective during periods of perinatal or postnatal nutrient insufficiency by prioritizing limited resources to the brain over less metabolically active peripheral tissues. This is supported by the evidence of increased glucose transport into the brain vs. the lung, liver, skeletal muscle, and heart in rat and sheep models of IUGR $(1,15,16)$. Glucose enters the brain down a concentration gradient via the Glut 1 facilitative transport protein on the blood brain barrier and is transported into neurons via Glut 3. Monocarboxylate transporters (MCTs) are found on the plasma and mitochondrial membrane of a variety of tissues, including the brain, and transport lactate, pyruvate, and ketone bodies. MCT1 is found on the blood brain barrier, neurons, glia, astrocytes, and retina, while MCT2 is expressed almost exclusively in postsynaptic neurons, having a higher affinity for substrates than MCT1 (ref. 17) and is essential for cerebral metabolism by coupling astrocyte and neuronal metabolism via the lactate-pyruvate shuttle (17). This preferential substrate transport along with increased cerebral blood flow and more efficient utilization of non-glucose substrates (i.e., lactate, ketone bodies, and amino acids) have been proposed as mechanisms behind the "brain-sparing" effect seen with asymmetric IUGR $(1,15,16)$. However, given the neurologic deficits described above, it is likely that the entire brain is not "spared" and that some regions such as the HPC or cerebral cortex may be more vulnerable to injury than others.

The objective of this study was to determine the vulnerability of the cerebral cortex and HPC of the developing IUGR rat brain to injury during acute insulin-induced HG. Our purpose was to understand the role of fetal metabolic adaptations and not to model the human scenario of HG in the IUGR neonate. We hypothesized that due to fetal metabolic adaptations the developing IUGR brain will be less vulnerable to injury from AHG than normally grown (NG) controls and that the regional distribution of injury will be preserved.

We determined neuronal injury after $4 \mathrm{~h}$ of AHG in the HPC and cortex of postnatal day (P)-14 IUGR and NG pups using Fluoro-Jade B (FJB) histochemistry. FJB stains for dead or stressed

'Department of Pediatrics, Division of Neonatology, University of Minnesota, Minneapolis, MN, USA. Correspondence: Anne M. Maliszewski-Hall (amalisze@umn.edu) 
cells and has been previously used to quantify HG-induced neuronal injury in developing and adult rats $(7,10,18)$. We studied the effects in P14 pups because of their neurodevelopmental similarities with the human term infant (19). We also measured fasting $\beta$-hydroxybutyrate (BHB) concentrations in blood and the transcript expression of glucose and MCTs (Glut 1, Glut 3, MCT1, and MCT2) in the cortex and HPC.

\section{RESULTS}

\section{Animal Weights}

Intrauterine growth restriction (IUGR) was induced via bilateral uterine artery ligation on gestational day (G) 19 as previously described (16). Body weights of the IUGR pups were $27 \%$ lower than the NG pups (IUGR: $4.5 \pm 0.01 \mathrm{~g}$ vs. NG: $5.9 \pm 0.01 \mathrm{~g}, P<0.001)$ at birth, and this effect persisted through P14 (IUGR: $29.8 \pm 0.8 \mathrm{~g}$ vs. NG: $32.7 \pm 0.5 \mathrm{~g}, P<0.01$ ). Brain weight measured at P14 did not differ between the groups (IUGR: $0.95 \pm 0.01 \mathrm{~g}$ vs. NG: $0.92 \pm 0.01 \mathrm{~g}$ ).

\section{Blood Glucose and $\beta$-Hydroxybutyrate (BHB) Concentrations}

We induced acute hypoglycemia (AHG) in NG and IUGR P14 rats using a combination of fasting and insulin administration (7). Littermates in the non-hypoglycemic control group $(\mathrm{CON})$ were similarly fasted and received normal saline yielding four experimental groups: IUGR/HG (IUGR and insulin), IUGR/CON (IUGR and saline), NG/HG (NG and insulin), and NG/CON (NG and saline). The control groups receiving normal saline (IUGR/CON and NG/CON) remained euglycemic (blood glucose $>50 \mathrm{mg} / \mathrm{dl}$ ) throughout the study period. After the overnight fast, blood glucose concentrations at baseline

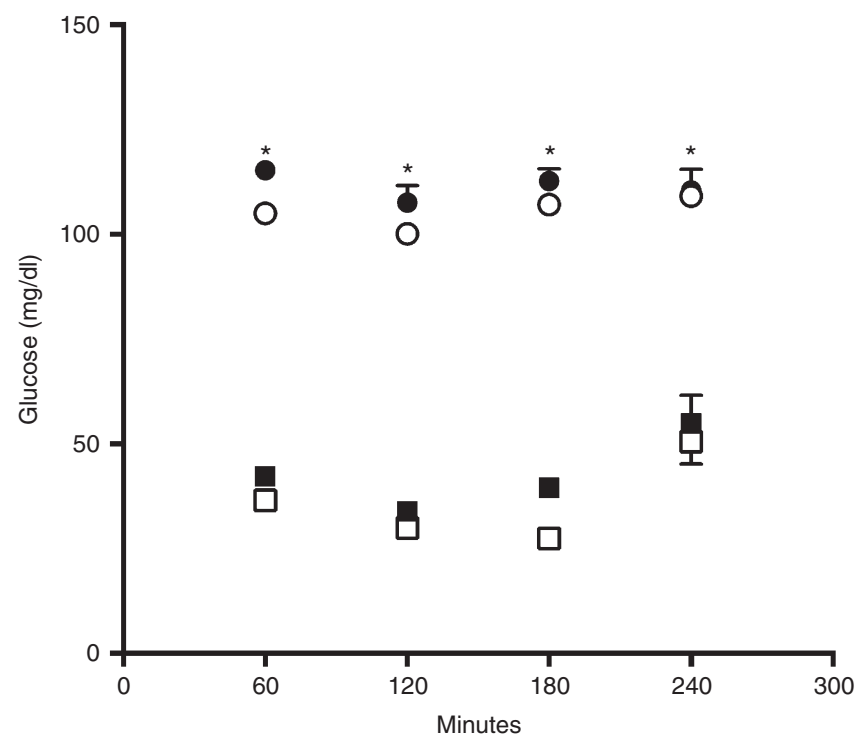

Figure 1. Blood glucose concentrations in control and hypoglycemia (HG) groups in normally grown (NG) and intrauterine growth restriction (IUGR) P14 rat pups. Blood glucose concentrations were lower in IUGR/ $\mathrm{HG}$ and NG/HG compared with NG/CON and IUGR/CON (treatment effect, $\left.{ }^{*} P<0.01\right)$. The mean blood glucose concentrations were similar between IUGR/HG and NG/HG. IUGR/CON, black circles; IUGR/HG, black squares; $\mathrm{NG} / \mathrm{CON}$, open circles; and NG/HG, open squares. Values are mean \pm SEM. $N=20-40$ rats/group at each time point. (time 0 ) were similar between groups (NG: $105.1 \pm 1.6 \mathrm{mg} / \mathrm{dl}$ vs. IUGR: $106.8 \pm 1.8 \mathrm{mg} / \mathrm{dl}$ ). Beginning $30 \mathrm{~min}$ after the administration of insulin and until the termination of HG 240 min after the insulin administration (i.e., for $210 \mathrm{~min}$ ), the mean blood glucose concentrations were lower in the IUGR/HG and NG/ HG groups (Figure 1). The severity of HG did not differ between the IUGR/HG and NG/HG groups. The blood glucose concentrations were measured in seven IUGR/HG and eight NG/HG pups $30 \mathrm{~min}$ after receiving the rescue dose of $10 \%$ dextrose (at $\sim 270 \mathrm{~min}$ ). There was no difference between the groups (IUGR: $124.7 \pm 14.9 \mathrm{mg} / \mathrm{dl}$ vs. NG: $159.3 \pm 21.6 \mathrm{mg} / \mathrm{dl}$ ). There was no difference in fasting BHB concentrations in NG vs. IUGR (NG: $1.24 \pm 0.05 \mathrm{mmol} / \mathrm{l}$ vs. IUGR: $1.45 \pm 0.11 \mathrm{mmol} / \mathrm{l})$.

Core Body Temperature (CBT) During Acute Hypoglycemia (AHG) Using a fiberoptic rectal probe, core body temperature (CBT) was measured every hour during the $4 \mathrm{~h}$ study period. The CBT was lower in IUGR/HG compared with the NG/HG $\left(31.94 \pm 0.01{ }^{\circ} \mathrm{C}\right.$ vs. $\left.33.58 \pm 0.06{ }^{\circ} \mathrm{C}, P<0.05\right)$.

\section{Neuronal Injury}

Neuronal injury was assessed $24 \mathrm{~h}$ after AHG using FJB histochemistry as in previous studies (7). There were rare FJBpositive (FJB+) cells in the cerebral cortex and HPC of the NG/ $\mathrm{CON}$ and IUGR/CON groups. FJB+ cells were present in the NG/HG and IUGR/HG groups, primarily in the cerebral cortex. The FJB+ cells were generally symmetrical between hemispheres and were isolated with staining of the cell body and neuronal processes. When compared with the NG/HG group, fewer FJB+ cells were present in the IUGR/HG group in the cerebral cortex $(P<0.01$, Figure 2$)$. There was no difference in

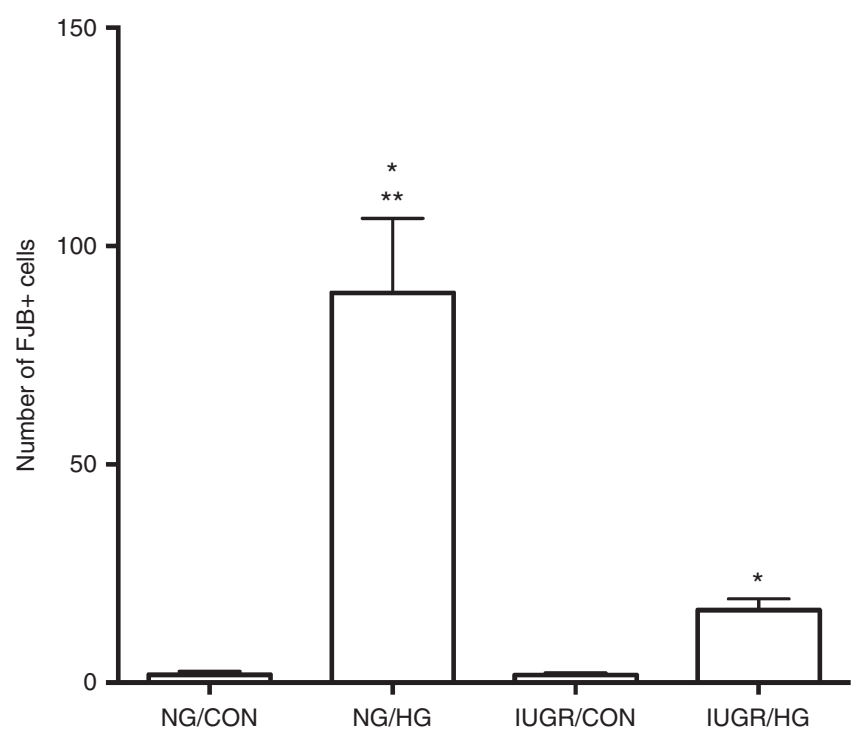

Figure 2. Fluoro-Jade B-positive (FJB+) cells in the cerebral cortex of P14 rat pups in the NG and IUGR groups at $24 \mathrm{~h}$ post-HG. The number of FJB+ cells in the IUGR/HG cortex was significantly lower compared with the number of FJB+ cells in the NG/HG cortex (group effect, ${ }^{* *} P<0.01$ ). The number of $\mathrm{FJB}+$ cells in the IUGR/HG and NG/HG cortex was elevated compared with IUGR/CON and NG/CON, respectively (treatment effect, ${ }^{*} P<0.05$ ). Values are mean \pm SEM FJB+ cells per brain section. $N=4-5$ rats/group. 
$\mathrm{FJB}+$ cells between NG/HG and IUGR/HG groups in the HPC (data not shown). To determine whether the FJB differences between the IUGR and NG group were due to differences in the neuronal number, Nissl histochemical analysis was performed. The intensity of Nissl staining did not differ between IUGR and NG in either the cortex or HPC (data not shown).

To further confirm that the differences in the severity of injury between IUGR/HG and NG/HG groups was not due to variations in the onset of injury, brain sections were obtained from subsets of animals in the IUGR/HG and NG/HG groups $6 \mathrm{~h}$ and $72 \mathrm{~h}$ post-HG and stained for FJB histochemistry. At both time points, IUGR/HG had fewer FJB+ cells in the cerebral cortex than NG/HG (Table 1). Similarly there was no difference in the number of FJB+ cells in the HPC at either time point.

There was no relationship between the number of FJB+ cells and blood ketone concentration, CBT, or postrecovery glucose concentration (data not shown).

\section{Substrate Transporter Transcript Expression}

The expression of Glut 1, Glut 3, MCT1, and MCT2 transcripts in the cortex and HPC was determined using quantitative PCR in P14 IUGR and NG pups under euglycemic conditions. The expression of MCT2 mRNA was significantly increased in the

Table 1. Fluoro-Jade B-positive (FJB+) cells in the cerebral cortex and hippocampus of IUGR and NG hypoglycemic rat pups

\begin{tabular}{llllll}
\hline & \multicolumn{2}{c}{ Cortex } & & \multicolumn{2}{c}{ Hippocampus } \\
\cline { 2 - 3 } \cline { 5 - 6 } & \multicolumn{1}{c}{$6 \mathrm{~h}$} & $72 \mathrm{~h}$ & & $6 \mathrm{~h}$ & $72 \mathrm{~h}$ \\
\hline IUGR/HG & $34.3 \pm 10.4^{*}$ & $13.4 \pm 2.0^{*}$ & & $3.0 \pm 0.6$ & $1.3 \pm 0.5$ \\
NG/HG & $77.0 \pm 9.3$ & $56.1 \pm 14.6$ & & $1.75 \pm 0.6$ & $3.8 \pm 0.9$ \\
\hline
\end{tabular}

Values are mean \pm SE. Number of FJB + cells in the region from four to six brain sections. For $6 h, n=4$ per group; for $72 h, n=7 \mathrm{IUGR} / \mathrm{HG}$ and $n=8 \mathrm{NG} / \mathrm{HG}$.

$\mathrm{HG}$, hypoglycemia; IUGR, intrauterine growth restriction; $N G$, normally grown. ${ }^{*} P<0.05$ for IUGR/HG vs. NG/HG.

\section{a}

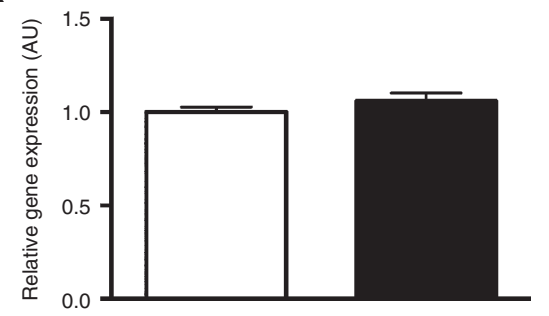

c

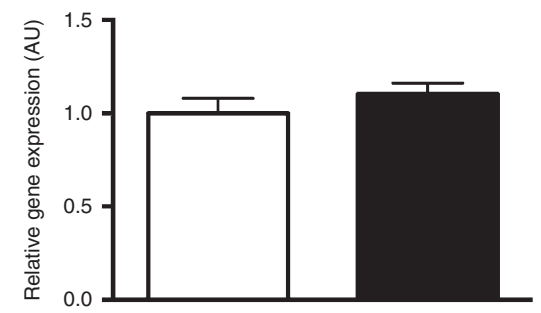

cerebral cortex of the IUGR group, relative to the NG group (Figure 3b). There was no difference in hippocampal MCT2 gene expression between the two groups (Figure $3 \mathrm{~d}$ ). There was no difference between the groups with respect to Glut 1, Glut 3, and MCT1 mRNA expression in either brain region (MCT1 Figure 3a,c; Glut data not shown, $P>0.25$ for all comparisons).

\section{DISCUSSION}

Asymmetric IUGR or growth restriction with head sparing is a growth pattern often seen as a result of placental insufficiency $(2,14,15)$. The mechanism of brain sparing is unknown; however, increased cerebral blood flow prioritizing essential nutrients and substrates to the brain at the expense of metabolically less active peripheral tissues, such as the liver and skeletal muscle may in part be responsible for this growth pattern $(1,2,15,20)$. Although brain size may be spared at birth, it is unknown whether the brain function is similarly spared and whether individual brain regions are protected equally during common metabolic perturbations in the postnatal period, such as HG. Understanding the effects of postnatal HG on the developing IUGR brain is clinically relevant since many IUGR neonates experience recurrent or prolonged episodes of $\mathrm{HG}$ well beyond the immediate newborn period (21-23).

Our objective in this study was to better understand how adaptations that result from IUGR might be protective or harmful in the setting of a metabolic insult such as AHG. We used a well-established rat model of placental insufficiency $(12,16)$ to determine whether the developing IUGR brain is more or less vulnerable to secondary injury from postnatal HG than NG rats. We chose P14 because our group has shown previously that the NG developing rat brain is resistant to neuronal injury from AHG until approximately P14 (ref. 7). Using FJB immunohistochemistry, we demonstrate that $4 \mathrm{~h}$ of acute,

b

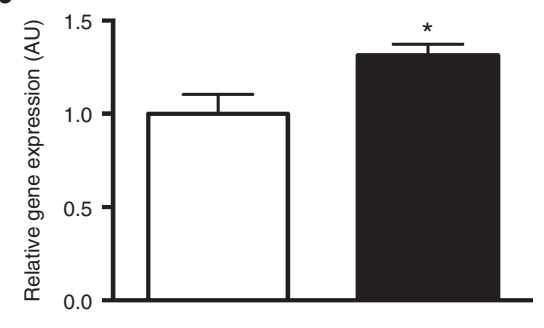

d

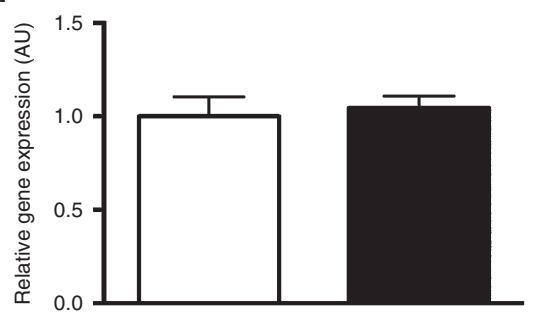

Figure 3. Monocarboxylate transporter (MCT) mRNA expression in P14 rat cortex and hippocampus (HPC) under basal conditions. NG, open box; IUGR, black box. (a) MCT1 gene expression in the cortex is similar between both groups. (b) MCT2 expression is elevated in the IUGR cortex $\left({ }^{*} P<0.03\right)$. Hippocampal MCT1 (c) and MCT2 (d) gene expression are similar in both groups. Values are mean \pm SEM relative gene expression in arbitrary units (AU). $N=6$ pups per group. 


\section{Articles | Maliszewski-Hall et al.}

insulin-induced HG leads to neuronal injury in the cerebral cortex of IUGR rats. However, compared with the NG rats, the injury was less severe. There was no difference in the severity of neuronal injury in the HPC. This is consistent with previous work from our group and others who have found an agedependent regional vulnerability to HG, such that the cerebral cortex is more vulnerable to neuronal damage than the striatum or $\operatorname{HPC}(7,10,11,24)$. The severity of injury as determined by the number of FJB+ cells in the cortex and HPC of NG/HG was similar to our previous reports $(7,25)$. Thus, IUGR protects the developing cortex during AHG, but does not alter the regional distribution of injury previously demonstrated in this model of AHG (7,10,11). Interestingly, CBT was lower in the IUGR pups and systemic hypothermia is known to be neuroprotective in many settings (26). Furthermore, increased MCT2 transcript expression in the IUGR cerebral cortex suggests that increased delivery of alternative substrates (lactate, pyruvate, and ketone bodies) into the cerebral cortex might also play a role in protecting against neuronal injury during AHG.

We defined HG as a blood glucose concentration of $<40 \mathrm{mg} /$ $\mathrm{dl}$, a value known to be associated with decreased brain glucose concentration in developing rats $(20,27)$. There was no difference in blood glucose concentrations after an overnight fast between the IUGR and NG groups. Furthermore, the sensitivity to insulin was similar in both the IUGR/HG and NG/ HG groups (Figure 1). Although studies employing a variety of IUGR animal models, including the bilateral uterine artery surgery used here, have shown fetal hypoglycemia and disrupted glucoregulation at birth $(1,3,14)$, the blood glucose concentrations and hepatic gluconeogenic enzyme activity normalize by $10 \mathrm{~d}$ of age in the rat (12), consistent with our findings of normal fasting blood glucose concentrations in P14 IUGR rat pups. Likewise, in our experimental groups receiving saline (IUGR/CON and NG/CON), there was no difference in blood glucose concentrations during the 4-h study period (Figure 1). This finding further supports that postnatal glucose metabolism and regulation are intact in IUGR.

However, despite normal fasting glucose concentrations and apparent equivalent insulin sensitivity at P14, our results show that the IUGR cerebral cortex is more resistant to injury than NG pups. The mechanisms underlying these observations currently remain unexplained. It has been demonstrated in animal and human studies that as a result of placental insufficiency, the fetus undergoes metabolic adaptations that promote survival in the short term, which may prove to be deleterious in the long term $(1,2,16,28)$. It is possible that these beneficial metabolic adaptations extend after birth, and the IUGR pup is able to more efficiently utilize glucose-sparing substrates such as ketones and lactate than NG pups. It is also possible that the energy requirement of the IUGR brain is low due to a decrease or delay in the ontogeny of energy-demanding developmental processes, such as synaptogenesis and myelination, after birth (29-31). Therefore brain injury might not occur when glucose concentrations are low.

To investigate the role of alternative substrates in IUGR further, we measured fasting blood $\mathrm{BHB}$ concentrations in both IUGR and NG prior to the administration of insulin. Although the difference in fasting BHB concentrations was not significant between the two groups, we found increased gene expression of MCT2 in the cortex of IUGR pups at baseline (non-fasting, euglycemic) compared with the NG pups suggesting that increased transport of alternative substrates prior to the onset of AHG might be protective against neuronal injury during glucose deprivation. Consistent with this possibility, previous studies have demonstrated that ketonemia, either innate or induced using a ketogenic diet, prevents neuronal injury during AHG in developing rats (18). Furthermore, increased expression of MCT2 mRNA has been shown in the brainstem of female rats in response to food deprivation, supporting increased utilization of ketone bodies as a respiratory fuel under nutrient restricted conditions (32). Other studies have reported increased MCT2 expression in response to noradrenalin (33) and insulin-like growth factor I and insulin via the mTOR pathway (34). In our study, there was no difference with respect to MCT1 in either the cerebral cortex or HPC (Figure 3a,c). The differences observed in our study between the expression of MCT1 and MCT2 might be due to the species-specific affinities of the MCTs. In rats, MCT1 and 2 are both expressed in neurons; however, MCT2 has a 5-10-fold higher affinity for pyruvate, lactate, acetoacetate, and BHB (17). Therefore, the magnitude of elevation in MCT2 here is likely to be clinically significant for this species. To our knowledge, this is the first study to report the effect of IUGR on MCT1 and MCT2 mRNA expression in the brain regions.

In addition to monocarboxylate transport, we also analyzed gene transcripts of the facilitative glucose transporters Glut 1 and Glut 3. Using a variety of animal models of IUGR, several groups have reported elevated (absolute and relative) brain Glut 1 expression prenatally and/or soon after birth, suggesting that this is an adaptation to prioritize glucose delivery to the fetal brain over peripheral tissues in IUGR $(1,15,16)$. We found no difference in Glut 1 or Glut 3 expression in either the cortex or HPC between IUGR and NG groups. This is not surprising given that our studies were performed at P14 when the pups were no longer hypoglycemic and had established normal feeding patterns. The similar plasma glucose concentration after overnight fasting in the two groups is consistent with this possibility. Sadiq et al. (15) in their study also found lower Glut 1 expression on P14, relative to the expression on $\mathrm{P} 1$, supporting postnatal recovery. Since the focus of our study was regional vulnerability of the brain to AHG, we did not measure peripheral glucose transport. Hence, it is unknown whether the IUGR pup continues to prioritize glucose centrally over the periphery on P14 and whether glucose metabolism has normalized in all organ systems.

Another possible explanation for our findings of reduced neuronal injury in the P14 IUGR cortex could be related to delayed neurodevelopment in IUGR. The developing rat brain has a very-low glucose utilization rate from birth until $\sim 10 \mathrm{~d}$ of life when it increases in a sigmoidal fashion (35). From P10-17, the rat begins to acquire audition, vision, play, and locomotion and this corresponds to an increase in glucose utilization rates in the brain regions subserving those functions $(36,37)$. Hence 
the demand of glucose by the brain region increases concurrently with its rapid phase of structural and functional development. In our previous study, we showed that postnatal age greatly affected the degree of neuronal injury after AHG in the NG developing rat such that prior to P14, there was no significant injury in any brain region (7). This suggests that as critical brain functions begin to develop, the demand for glucose increases and, when the supply cannot meet the demand, the brain is at a higher risk for injury. To what extent IUGR delays brain maturation is not well understood; however, functional neurodevelopmental delays have been noted in infants and children who were born IUGR (4). Furthermore, other studies investigating the effect of fetal nutritional deficiencies on neurodevelopment have shown shifts in the critical period to a later time point along the developmental axis (38). Specifically, in perinatal iron-deficient rat pups, the critical window of hippocampal development is later and shorter than in iron-sufficient pups. This is also associated with lower plasticity and altered long-term potentiation in the adult, formerly iron-deficient rat (38). Therefore, it is plausible that the reduced neuronal injury observed after AHG in IUGR may in fact be related to a lower demand for glucose at that developmental age due to a delay in neurodevelopment. This is further supported by the observations by Ogata et al. (12) in P21 IUGR rats where $48 \mathrm{~h}$ of fasting resulted in significant reductions in plasma glucose, insulin, and alanine concentrations and elevated BHB concentrations, suggesting that the period for peak cerebral metabolism and glucose demand is offset in IUGR occurring closer to P21 and outside of the normal P10-17 window. The cerebral cortex may particularly benefit from this developmental shift as cortical development peaks later than all other brain regions (39).

One novel observation in this study is that the IUGR/HG pups were noted to have systemic hypothermia during the study period. Although ambient temperature was controlled uniformly between the two groups, IUGR CBTs were significantly lower than NG at baseline and throughout the duration of the study. The average CBT for the IUGR/HG group was $31.94 \pm 0.01{ }^{\circ} \mathrm{C}$ compared with $33.58 \pm 0.06{ }^{\circ} \mathrm{C}$ in the NG/HG. Central hypothermia $\left(\mathrm{CBT}<34^{\circ} \mathrm{C}\right.$ ) is known to be neuroprotective in both neonates and adults after severe hypoxic insults. Although it is unclear from this study to the extent at which central hypothermia may provide neuroprotection, it might also be indicative of a lower resting metabolism seen as a protective adaptation to IUGR. Interestingly, the control group (NG/HG) had an average $\mathrm{CBT}<34^{\circ} \mathrm{C}$ yet still displayed significant injury in the cortex. When analyzed using linear regression, there was no relationship between CBT and number of FJB+ cells. The exact role of hypothermia in this setting needs to be explored further.

Our study has some limitations. We used Nissl histochemical analysis of limited number of brain sections to determine neuronal structure and number in the NG and IUGR groups, instead of the stereologic assessments. Our group has previously demonstrated that Nissl histochemical assessment is a valid method for assessing neuronal density after hypoxia-ischemia (40).
Another limitation of this study is that we only included follow-up through $72 \mathrm{~h}$ and therefore cannot rule out late injury occurring beyond that time point. However, our group has shown that peak neuronal injury after AHG occurs at or before $24 \mathrm{~h}$ (7). Further, we observed similar patterns and severity of injury at 6,24 , and $72 \mathrm{~h}$ post-HG in IUGR/HG vs. NG/HG suggesting variations in the onset of injury in the NG and IUGR groups as the cause for the discrepant results is unlikely (Figure 2, Table 1). Lastly, we did not evaluate long-term functional or behavioral studies in this pilot study. Functional outcomes would greatly strengthen the clinical significance of our findings.

In summary, using a rodent model of IUGR we found that the cerebral cortex of the IUGR rat pup is less vulnerable to neuronal injury during postnatal HG than NG controls. We also found a significant reduction in the CBT and increased gene expression of the neuronal MCT2 in the cerebral cortex of the IUGR rats. We speculate that adaptations such as systemic hypothermia or enhanced production and delivery of alternative substrates to the cerebral cortex might be neuroprotective in the setting of postnatal metabolic perturbations in IUGR.

\section{METHODS}

\section{Animal Preparation}

Timed-pregnant Sprague-Dawley rats were received on day 13-15 of gestation (Harlan Laboratories, Madison, WI) and individually housed under standard laboratory conditions with free access to standard rat chow and water. On gestational (G) day 19 (term 22 d), the pregnant rats were anesthetized with inhaled isoflurane (1.5-3\%) in a 50:50 mixture of $\mathrm{N}_{2} \mathrm{O}$ and $\mathrm{O}_{2}$, and both uterine arteries were ligated as previously described $(12,16)$. Normally grown (NG) control animals did not undergo surgery since previous studies have shown no differences in metabolic phenotype between sham-operated control group and non-operated controls after P4 (refs. 12 and 16). Rats recovered within an hour of surgery and were provided standard postoperative care. Dams were allowed to deliver spontaneously. Pups were weighed within $24 \mathrm{~h}$ of birth, the four largest animals were killed, and litters were culled to a maximum of eight. Dams and pups were housed in a temperature- and humidity-controlled animal care facility with 12 $\mathrm{h}: 12 \mathrm{~h}$ light:dark cycle and allowed food and water ad libitum. All experiments were conducted in accordance with the principles and procedures outlined in the National Institutes of Health Guide for the Care and Use of Laboratory Animals. The Institutional Animal Care and Use Committee at the University of Minnesota approved all experimental protocols.

\section{Induction of Hypoglycemia (HG)}

On postnatal day $(\mathrm{P}) 14$, the IUGR and NG rats were subjected to a single episode of AHG as previously described (7). In brief, AHG was induced using a $6 \mathrm{IU} / \mathrm{kg}$ intraperitoneal (i.p) injection of human regular insulin (Novo Norkisk, Clayton, NC) after a period of overnight fasting. The target blood glucose was $<40 \mathrm{mg} / \mathrm{dl}$. Four experimental groups were created including NG control pups, which remained euglycemic (NG/CON), NG pups with HG (NG/HG), and IUGR/CON and IUGR/HG. Littermates in the CON groups were similarly fasted and were subcutaneously (s.c.) injected with an equivalent volume of $0.9 \%$ saline. The glucose concentrations were measured in whole blood collected from tail vein at baseline using a blood glucose meter (Accu-Check Compact, Roche Diagnostics, Indianapolis, IN). The whole-blood $\mathrm{BHB}$ concentration was measured at $0 \mathrm{~min}$ using a blood $\beta$-ketone monitoring system (Precision Xtra, Abbott Laboratories, Oxon, UK). The blood glucose concentrations were determined every $30 \mathrm{~min}$ in the HG groups and every $60 \mathrm{~min}$ in the CON groups throughout the duration of the study. If blood glucose concentrations dropped below $20 \mathrm{mg} / \mathrm{dl}, 10 \%$ dextrose was administered in a dose of 
$200 \mathrm{mg} / \mathrm{kg}$, i.p. and s.c. in order to prevent seizures (7). No pups in either group experienced seizure activity throughout the study. AHG was terminated $240 \mathrm{~min}$ after the insulin administration by injecting $200 \mathrm{mg} / \mathrm{kg}$ of $10 \%$ dextrose i.p. and s.c. before reuniting the pups with their respective dams. The control pups not receiving insulin were similarly injected with an equal volume of $0.9 \%$ normal saline. Postrescue glucose concentrations were measured $30 \mathrm{~min}$ after the administration of $10 \%$ dextrose. In a subset of IUGR/HG and NG/HG pups, rectal temperature was measured every hour during AHG using a Fiberoptic Rectal Temperature Module (SA Instruments, Stony Brook, NY).

\section{Histochemical Analysis}

Histochemistry was performed as previously described (7) using FJB immunohistochemistry (Abcam, Cambridge, MA). In brief, brains were harvested 6, 24, and $72 \mathrm{~h}$ after the conclusion of AHG. Rats were deeply anesthetized with sodium pentobarbital $(100 \mathrm{mg} / \mathrm{kg}$, i.p.) before undergoing in situ transcardial perfusion with $0.9 \%$ saline followed by $4 \%$ formaldehyde and 5\% sucrose in PBS. Brains were removed and postfixed overnight at $4{ }^{\circ} \mathrm{C}$ in $4 \%$ formalin, followed by serial overnight passages in 20 and $30 \%$ sucrose in PBS at $4{ }^{\circ} \mathrm{C}$ for cryoprotection. Brains were then flash-frozen on dry ice and acetone bath and embedded in a tissue-freezing medium (Triangle Biomedical Sciences, Durham, NC) for sectioning. Serial $20-\mu \mathrm{m}$ coronal sections were obtained using a cryostat (Model CM1900; Leica Instruments GmbH, Nussloch, Germany) at $-22{ }^{\circ} \mathrm{C}$. FJB staining and analysis were performed as described in our previous study (7). All FJB+ cells in the brain regions of each brain section were counted and group means were determined. To assess differences in neuronal structure and cellular intensity, Nissl staining was performed as previously described (40).

\section{Quantitative PCR (qPCR)}

Total RNA was isolated from dissected HPC and cortex using an RNAisolation kit (Life Technologies, Grand Island, NY) and was used to generate cDNA by reverse transcription. All qPCR experiments were performed with $4 \mu \mathrm{l}$ of diluted cDNA, $5 \mu \mathrm{l}$ FastStart Universal Probe Master Mix (Roche Diagnostics) and $0.5 \mu \mathrm{LEPC} \mathrm{H}_{2} \mathrm{O}$, and $0.5 \mu \mathrm{l} 20 \times$ Taqman Gene Expression Assay primer/probe (Agilent Technologies, Santa Clara, CA).

\section{Statistical Analysis}

Group means were compared using ANOVA with group (NG/CON, NG/HG, IUGR/CON, and IUGR/HG) and time of assessment (6, 24 , and $72 \mathrm{~h}$ ) as fixed factors. Intergroup differences were determined using unpaired $t$-tests. Data are presented as mean \pm SEM. Significance was set at $P<0.05$. The relationship between CBT and number FJB+ cells, ketone concentration and number of FJB+cells, and postrescue glucose concentration and number of FJB+ cells was analyzed by linear regression.

\section{ACKNOWLDEGMENTS}

A.M.M.-H. contributed to the experimental design, execution, interpretation, analysis, and manuscript preparation, A.B.S. and K.E. contributed to the execution, analysis, and manuscript preparation, M.A. contributed to execution and manuscript preparation, and R.R. contributed to experimental design, execution, interpretation, analysis, and manuscript preparation. The authors thank Michael Georgieff for critical review of the manuscript and Rebecca Simmons for technical and intellectual support.

\section{STATEMENT OF FINANCIAL SUPPORT}

The National Institutes of Health (CHRCDA K12 HD068322; Bethesda, MD) and the Viking Children's Fund, Department of Pediatrics, University of Minnesota, Minneapolis, MN, supported this project.

Disclosure: The authors have no financial ties to products in the study or potential/perceived conflicts of interest and therefore have nothing to disclose.

\section{REFERENCES}

1. Limesand SW, Rozance PJ, Smith D, Hay WW Jr. Increased insulin sensitivity and maintenance of glucose utilization rates in fetal sheep with placental insufficiency and intrauterine growth restriction. Am J Physiol Endocrinol Metab 2007;293:E1716-25.

2. Thorn SR, Rozance PJ, Brown LD, Hay WW Jr. The intrauterine growth restriction phenotype: fetal adaptations and potential implications for later life insulin resistance and diabetes. Semin Reprod Med 2011; 29:225-36.

3. Hayakawa M, Sato Y, Hattori T, et al. Carbohydrate and energy metabolism in the brain of rats with thromboxane A2-induced fetal growth restriction. Pediatr Res 2011;70:21-4.

4. Leitner Y, Fattal-Valevski A, Geva R, et al. Neurodevelopmental outcome of children with intrauterine growth retardation: a longitudinal, 10-year prospective study. J Child Neurol 2007;22:580-7.

5. Baschat AA. Neurodevelopment following fetal growth restriction and its relationship with antepartum parameters of placental dysfunction. Ultrasound Obstet Gynecol 2011;37:501-14.

6. Georgieff MK. Intrauterine growth retardation and subsequent somatic growth and neurodevelopment. J Pediatr 1998;133:3-5.

7. Ennis K, Tran PV, Seaquist ER, Rao R. Postnatal age influences hypoglycemia-induced neuronal injury in the rat brain. Brain Res 2008;1224:119-26.

8. Kirchhoff BA, Lugar HM, Smith SE, et al. Hypoglycaemia-induced changes in regional brain volume and memory function. Diabet Med 2013;30: e151-6.

9. Kim M, Yu ZX, Fredholm BB, Rivkees SA. Susceptibility of the developing brain to acute hypoglycemia involving A1 adenosine receptor activation. Am J Physiol Endocrinol Metab 2005;289:E562-9.

10. Moore H, Craft TK, Grimaldi LM, Babic B, Brunelli SA, Vannucci SJ. Moderate recurrent hypoglycemia during early development leads to persistent changes in affective behavior in the rat. Brain Behav Immun 2010;24:839-49.

11. Yamada KA, Rensing N, Izumi Y, et al. Repetitive hypoglycemia in young rats impairs hippocampal long-term potentiation. Pediatr Res 2004;55:372-9.

12. Ogata ES, Bussey ME, LaBarbera A, Finley S. Altered growth, hypoglycemia, hypoalaninemia, and ketonemia in the young rat: postnatal consequences of intrauterine growth retardation. Pediatr Res 1985;19:32-7.

13. Hawdon JM. Neonatal metabolic adaptation after preterm delivery or intra-uterine growth retardation. Biochem Soc Trans 1998;26:123-5.

14. Lin CH, Gelardi NL, Cha CJ, Oh W. Cerebral metabolic response to hypoglycemia in severe intrauterine growth-retarded rat pups. Early Hum Dev 1998;51:147-57.

15. Sadiq HF, Das UG, Tracy TF, Devaskar SU. Intra-uterine growth restriction differentially regulates perinatal brain and skeletal muscle glucose transporters. Brain Res 1999;823:96-103.

16. Simmons RA, Gounis AS, Bangalore SA, Ogata ES. Intrauterine growth retardation: fetal glucose transport is diminished in lung but spared in brain. Pediatr Res 1992;31:59-63.

17. Halestrap AP. Monocarboxylic acid transport. Compr Physiol 2013;3: 1611-43.

18. Yamada KA, Rensing N, Thio LL. Ketogenic diet reduces hypoglycemiainduced neuronal death in young rats. Neurosci Lett 2005;385:210-4.

19. Romijn HJ, Hofman MA, Gramsbergen A. At what age is the developing cerebral cortex of the rat comparable to that of the full-term newborn human baby? Early Hum Dev 1991;26:61-7.

20. Vannucci RC, Vannucci SJ. Hypoglycemic brain injury. Semin Neonatol 2001;6:147-55.

21. Collins JE, Leonard JV, Teale D, et al. Hyperinsulinaemic hypoglycaemia in small for dates babies. Arch Dis Child 1990;65:1118-20.

22. Haymond MW, Karl IE, Pagliara AS. Increased gluconeogenic substrates in the small-for-gestational-age infant. N Engl J Med 1974;291:322-8.

23. Lubchenco LO, Bard H. Incidence of hypoglycemia in newborn infants classified by birth weight and gestational age. Pediatrics 1971;47:831-8.

24. Haces ML, Montiel T, Massieu L. Selective vulnerability of brain regions to oxidative stress in a non-coma model of insulin-induced hypoglycemia. Neuroscience 2010;165:28-38.

25. Rao R, Sperr D, Ennis K, Tran P. Postnatal age influences hypoglycemiainduced poly(ADP-ribose) polymerase-1 activation in the brain regions of rats. Pediatr Res 2009;66:642-7.

26. Karnatovskaia LV, Wartenberg KE, Freeman WD. Therapeutic hypothermia for neuroprotection: history, mechanisms, risks, and clinical applications. Neurohospitalist 2014;4:153-63.

27. Rao R, Ennis K, Long JD, Ugurbil K, Gruetter R, Tkac I. Neurochemical changes in the developing rat hippocampus during prolonged hypoglycemia. J Neurochem 2010;114:728-38. 
28. Simmons RA, Templeton LJ, Gertz SJ. Intrauterine growth retardation leads to the development of type 2 diabetes in the rat. Diabetes 2001;50:2279-86.

29. Bisignano M, Rees S. The effects of intrauterine growth retardation on synaptogenesis and mitochondrial formation in the cerebral and cerebellar cortices of fetal sheep. Int J Dev Neurosci 1988;6:453-60.

30. Reid MV, Murray KA, Marsh ED, Golden JA, Simmons RA, Grinspan JB. Delayed myelination in an intrauterine growth retardation model is mediated by oxidative stress upregulating bone morphogenetic protein 4 . J Neuropathol Exp Neurol 2012;71:640-53.

31. Schober ME, McKnight RA, Yu X, Callaway CW, Ke X, Lane RH. Intrauterine growth restriction due to uteroplacental insufficiency decreased white matter and altered NMDAR subunit composition in juvenile rat hippocampi. Am J Physiol Regul Integr Comp Physiol 2009;296:R681-92.

32. Matsuyama S, Ohkura S, Iwata K, et al. Food deprivation induces monocarboxylate transporter 2 expression in the brainstem of female rat. J Reprod Dev 2009;55:256-61.

33. Chenal J, Pellerin L. Noradrenaline enhances the expression of the neuronal monocarboxylate transporter MCT2 by translational activation via stimulation of PI3K/Akt and the mTOR/S6K pathway. J Neurochem 2007;102:389-97.

34. Chenal J, Pierre K, Pellerin L. Insulin and IGF-1 enhance the expression of the neuronal monocarboxylate transporter MCT2 by translational activation via stimulation of the phosphoinositide 3-kinase-Akt-mammalian target of rapamycin pathway. Eur J Neurosci 2008;27:53-65.

35. Nehlig A. Cerebral energy metabolism, glucose transport and blood flow: changes with maturation and adaptation to hypoglycaemia. Diabetes Metab 1997;23:18-29.

36. Nehlig A, de Vasconcelos AP, Boyet S. Quantitative autoradiographic measurement of local cerebral glucose utilization in freely moving rats during postnatal development. J Neurosci 1988;8:2321-33.

37. Nehlig A, Pereira de Vasconcelos A. Glucose and ketone body utilization by the brain of neonatal rats. Prog Neurobiol 1993;40:163-221.

38. Callahan LS, Thibert KA, Wobken JD, Georgieff MK. Early-life iron deficiency anemia alters the development and long-term expression of parvalbumin and perineuronal nets in the rat hippocampus. Dev Neurosci 2013;35:427-36.

39. Rice D, Barone S Jr. Critical periods of vulnerability for the developing nervous system: evidence from humans and animal models. Environ Health Perspect 2000;108:Suppl 3:511-33.

40. Rao R, Tkac I, Townsend EL, Ennis K, Gruetter R, Georgieff MK. Perinatal iron deficiency predisposes the developing rat hippocampus to greater injury from mild to moderate hypoxia-ischemia. J Cereb Blood Flow Metab 2007;27:729-40. 